\title{
AQP9 promotes astrocytoma cell invasion and motility via the AKT pathway
}

\author{
YAO LV ${ }^{1}$, QIANG HUANG ${ }^{1}$, WEIMIN DAI ${ }^{1}$, YUANQING JIE ${ }^{1}$, GUOFENG YU ${ }^{1}$, \\ XIAOFENG FAN $^{1}$, AN WU $^{1}$ and QIAN MIAO ${ }^{2}$
}

Departments of ${ }^{1}$ Neurosurgery and ${ }^{2}$ Oncology, Quzhou People's Hospital, Quzhou, Zhejiang 324000, P.R. China

Received August 25, 2016; Accepted March 7, 2018

DOI: $10.3892 / \mathrm{ol} .2018 .9361$

\begin{abstract}
The aquaporin (AQP) family, which includes 13 members identified in mammalian cells, is involved in cancer development and progression. AQP9 expression is upregulated in several tumor tissue types. However, the functions of AQP9 in astrocytoma remain elusive. The present study identified that AQP9 was expressed in astrocytoma cells. AQP9 expression was silenced by transfection with small interfering RNAs and increased by transfection with a plasmid containing the AQP9 gene. Using invasion and wound-healing assays, it was revealed that the knockdown of AQP9 suppressed astrocytoma cell invasion and motility, whereas overexpression of AQP9 promoted the invasion and motility of astrocytoma cells. It was further revealed that AQP9 could induce RAC serine/threonine-protein kinase (AKT) activation and decrease E-cadherin expression in astrocytoma cells. Inhibition of the AKT pathway attenuated AQP9-mediated invasion, motility and E-cadherin expression. Taken together, the results of the present study indicated that AQP9 promoted the invasion and motility of cells via the AKT pathway. Therefore, AQP9 may represent a potential target for therapeutic use of astrocytoma.
\end{abstract}

\section{Introduction}

Astrocytoma is one of the most common primary brain tumors, and is one of the leading causes of cancer-associated mortality worldwide (1). Despite the great progression in brain tumor therapy, invasion and metastasis remain the major reasons for the poor survival rate of patients with astrocytoma (2). Therefore, uncovering the molecular mechanism of astrocytoma cell invasion is urgently required and necessary.

Correspondence to: Dr Qian Miao, Department of Oncology, Quzhou People's Hospital, 2 Zhong Loudi Road, Quzhou, Zhejiang 324000, P.R. China

E-mail: miaoqiann12@163.com

Key words: AQP9, astrocytoma, invasion, motility, RAC serine/threonine-protein kinase, E-cadherin
Aquaporins (AQPs), a family of small membrane transport proteins, aid the transportation of water and small solutes such as glycerol (3). There are 13 AQPs that have been identified in mammalian cells, which can be divided into two groups. One group, which selectively transports water alone, includes AQP1, $2,4,5$, and 8 . The other group can transport water and other small solutes, and includes AQP3, 7, 9, and 10 (4). Studies have revealed that several AQPs are overexpressed in tumors $(5,6)$, and thus serve notable roles in tumor progression. A previous study also revealed that AQP3 promotes prostate cancer cell invasion via extracellular signal-regulated kinase 1/2-mediated matrix metalloproteinase-3 secretion (7), whereas AQP4 can enhance cell invasion in human glioma cells (8). It was reported that AQP9 was upregulated in human glioma tissues (9), and AQP9 expression was closely associated with the pathological grade of astrocytic tumors (10). However, the molecular functions of AQP9 in astrocytoma cells remain elusive.

The present study revealed that AQP9 was expressed in astrocytoma cells. The current study also investigated the role of AQP9 in astrocytoma cell invasion and motility, and demonstrated that AQP9 enhanced astrocytoma cell invasion and motility via the RAC serine/threonine-protein kinase (AKT) pathway.

\section{Materials and methods}

Cell lines and culture conditions. The astrocytoma U251 and SHG44 cells were obtained from the Type Culture Collection of the Chinese Academy of Sciences (Shanghai, China). U251 cells were cultured in RPMI-1640 containing $10 \%$ fetal bovine serum (FBS), whereas SHG44 cells were cultured in DMEM (Gibco; Thermo Fisher Scientific, Inc., Waltham, MA, USA) containing $10 \%$ FBS. All cells were incubated at $37^{\circ} \mathrm{C}$ in a $5 \% \mathrm{CO}_{2}$ atmosphere.

Reagents. The antibodies against AQP9 (cat. no. sc-74409), AKT (cat. no. sc-24500), E-cadherin (cat. no. sc-71009) and $\beta$-actin (cat. no. sc-47778) were all purchased from Santa Cruz Biotechnology, Inc. (Dallas, TX, USA). The antibody against phosphorylated (p)-AKT (cat. no. 5012) was obtained from Cell Signaling Technology, Inc. (Danvers, MA, USA). A phosphoinositide 3-kinase (PI3K) specific inhibitor, LY294002, was purchased from Sigma-Aldrich; Merck KGaA (Darmstadt, Germany). 
Reverse transcription-quantitative polymerase chain reaction (RT-qPCR). RNA was isolated from cell lines using TRIzol reagent (Invitrogen; Thermo Fisher Scientific, Inc.). Next, cDNA was obtained by using the Omniscript Reverse Transcription kit (Qiagen, Inc., Valencia, CA, USA). qPCR was performed using SYBR-green master mix (Applied Biosystems, Thermo Fisher Scientific, Inc.), cDNA and primers of AQP9 and $\beta$-actin (AQP9 forward, 5'-GAAGAG CAGCTTAGCGAAAGA-3' and reverse, 5'-ACAGCCACA TCCAAGGACAAT-3'; and $\beta$-actin forward, 5'-ATAGCA CAGCCTGGATAGCAACGTAC-3' and reverse, 5'-CACCTT CTACAATGAGCTGCGTGTG-3'). The real-time PCR reaction was processed as: $95^{\circ} \mathrm{C}$ for $10 \mathrm{~min}$, followed by 40 cycles of $95^{\circ} \mathrm{C}$ for $15 \mathrm{sec}$, and $60^{\circ} \mathrm{C}$ for $50 \mathrm{sec}$. Finally, the mRNA level of AQP9 was normalized to $\beta$-actin expression, and were calculated based on the $2^{-\Delta \Delta \mathrm{Cq}}$ method (11).

Western blot analysis. Cells were lysed in radioimmunoprecipitation assay lysis buffer $(0.05 \mathrm{M}$ Tris- $\mathrm{HCl}, \mathrm{pH} 7.4,0.15 \mathrm{M}$ $\mathrm{NaCl}, 0.25 \%$ deoxycholic acid, $1 \% \mathrm{NP}-40,1 \mathrm{mM}$ EDTA, $0.5 \mathrm{mM}$ dithiothreitol) containing protease and phosphatase inhibitors (Applygen Technologies, Inc., Beijing, China) to obtain total protein. A Bicinchoninic Acid Assay kit (Applygen Technologies, Inc.) was used to measure the total protein concentrations. Next, $50 \mu \mathrm{g}$ of protein was loaded and separated by $10 \%$ SDS-PAGE and electroblotted onto polyvinylidene difluoride membranes. The membranes were blocked in 5\% non-fat milk (for detection of AQP9, E-cadherin, $\beta$-actin and AKT) or 5\% bovine serum albumin (Sigma-Aldrich, Merck KGaA) (for detection of p-AKT). Next, the membranes were incubated with primary antibodies against AQP9 (1:500), AKT (1:1,000), p-AKT (1:1,000), E-cadherin (1:500) and $\beta$-actin $(1: 1,000)$ overnight at $4^{\circ} \mathrm{C}$. Then the membranes were incubated with the secondary antibodies (anti-mouse IgG, cat no. A0168 or anti-rabbit IgG, cat no. SAB3700863; Sigma-Aldrich, Merck KGaA) for $1 \mathrm{~h}$ at room temperature. Immunoreactive bands were visualized using an enhanced chemiluminescence kit (Applygen Technologies, Inc.). The band density was quantified with Quantity One 4.5.0 software (Bio-Rad Laboratories, Inc., Hercules, CA, USA), and $\beta$-actin was served as an internal control.

Cell transfection. To deplete AQP9 expression, small interfering RNA (siRNA) targeting AQP9 (siAQP9: 5'-GUC CGUAUCUCUACUAGCU-3') was obtained from Shanghai GenePharma Co., Ltd. (Shanghai, China) and a scramble siRNA was used as control siRNA (siCtrl: 5'-UUCUCC GAACGUGUCACUU-3'). To increase AQP9 expression, pcDNA3.1-AQP9 (pcAQP9) was obtained from Shanghai GenePharma Co., Ltd. and transfected into SHG44 cells; a control pcDNA3.1 (pcCtrl) was also used. Cells were transfected with siRNA $(50 \mathrm{nM})$ or plasmid $(800 \mathrm{ng} / \mu \mathrm{l})$ using Lipofectamine 2000 (Invitrogen; Thermo Fisher Scientific, Inc.). Then the cells were incubated for another $24 \mathrm{~h}$ and subjected to further investigation.

Cell invasion assay. A 24-well Transwell plate (Costar; Corning Life Sciences, San Diego, CA, USA) containing PET membranes with $8-\mu \mathrm{m}$ pores used to perform a cell invasion assay. In brief, the upper chambers of Transwell plate were
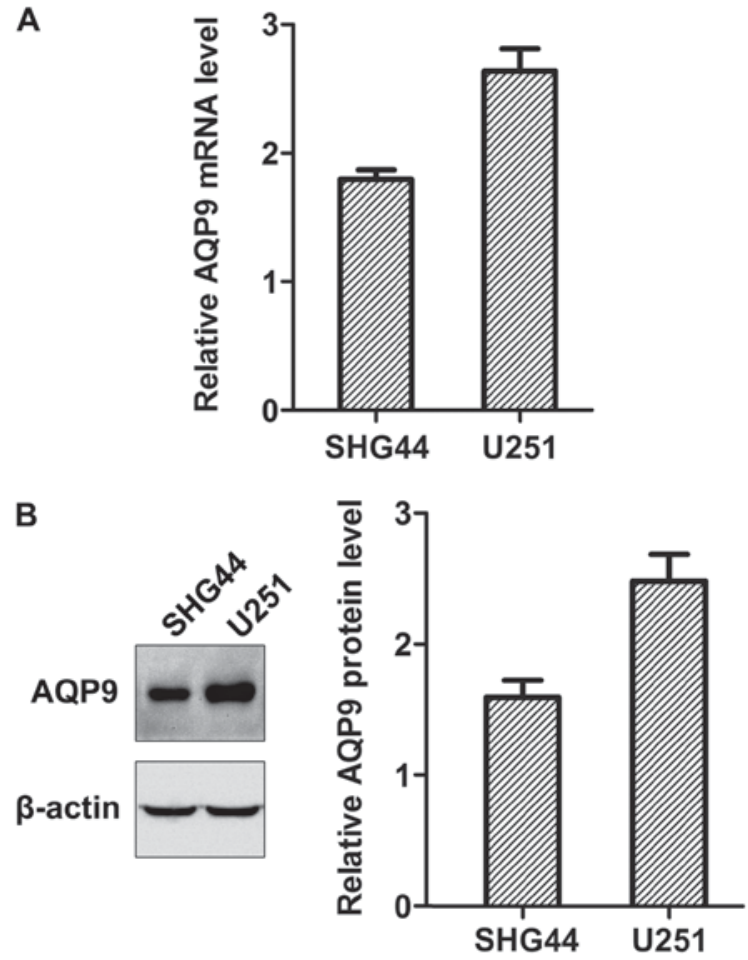

Figure 1. Expression of AQP9 in astrocytoma cells. (A) AQP9 mRNA expression in SHG44 and U251 cells was examined using reverse transcription-quantitative polymerase chain reaction. (B) AQP9 protein expression in SHG44 and U251 cells was examined using western blot analysis. AQP9, aquaporin 9 .

coated with Matrigel (BD Biosciences, Franklin Lakes, NJ, USA) for $30 \mathrm{~min}$. Next, $5 \times 10^{4}$ cells (U251 or SHG44 cells) in $200 \mu 1$ DMEM were loaded into the upper chambers, whereas $500 \mu \mathrm{l}$ of DMEM containing 30\% FBS were loaded into the lower chambers. Cells were pretreated with or without $10 \mu \mathrm{M}$ LY294002 for $30 \mathrm{~min}$ at $37^{\circ} \mathrm{C}$, and then the cells were allowed to invade for $24 \mathrm{~h}$ in a $\mathrm{CO}_{2}$ incubator at $37^{\circ} \mathrm{C}$, and then were fixed with $4 \%$ formaldehyde for $30 \mathrm{~min}$ at room temperature, and then stained with $0.5 \%$ crystal violet for $15 \mathrm{~min}$ at room temperature. The number of cells was counted in seven randomly selected fields under a light microscope at x200 magnification.

Wound-healing assay. U251 or SHG44 cells $\left(6 \times 10^{6}\right.$ cells $\left./ \mathrm{ml}\right)$ were seeded onto the 6 -well plate, and then incubated at $37^{\circ} \mathrm{C}$ for $12 \mathrm{~h}$. Next, wounds were made by a $200-\mu \mathrm{l}$ pipette tip. Images were captured at 0 and $24 \mathrm{~h}$ and the relative distance at the front was measured.

Statistical analysis. All experiments were performed at least three times, and all data were presented as the mean \pm standard deviation. Student's t-test was used to compare the significance between two groups, whereas one-way analysis with post hoc contrasts using the Student-Newman-Keuls test was used to compare the significance among multiple groups. $\mathrm{P}<0.05$ was considered to indicate a statistically significant difference.

\section{Results}

AQP9 is expressed in astrocytoma cells. The AQP9 mRNA levels in SHG44 and U251 cells were detected by RT-qPCR. 
A
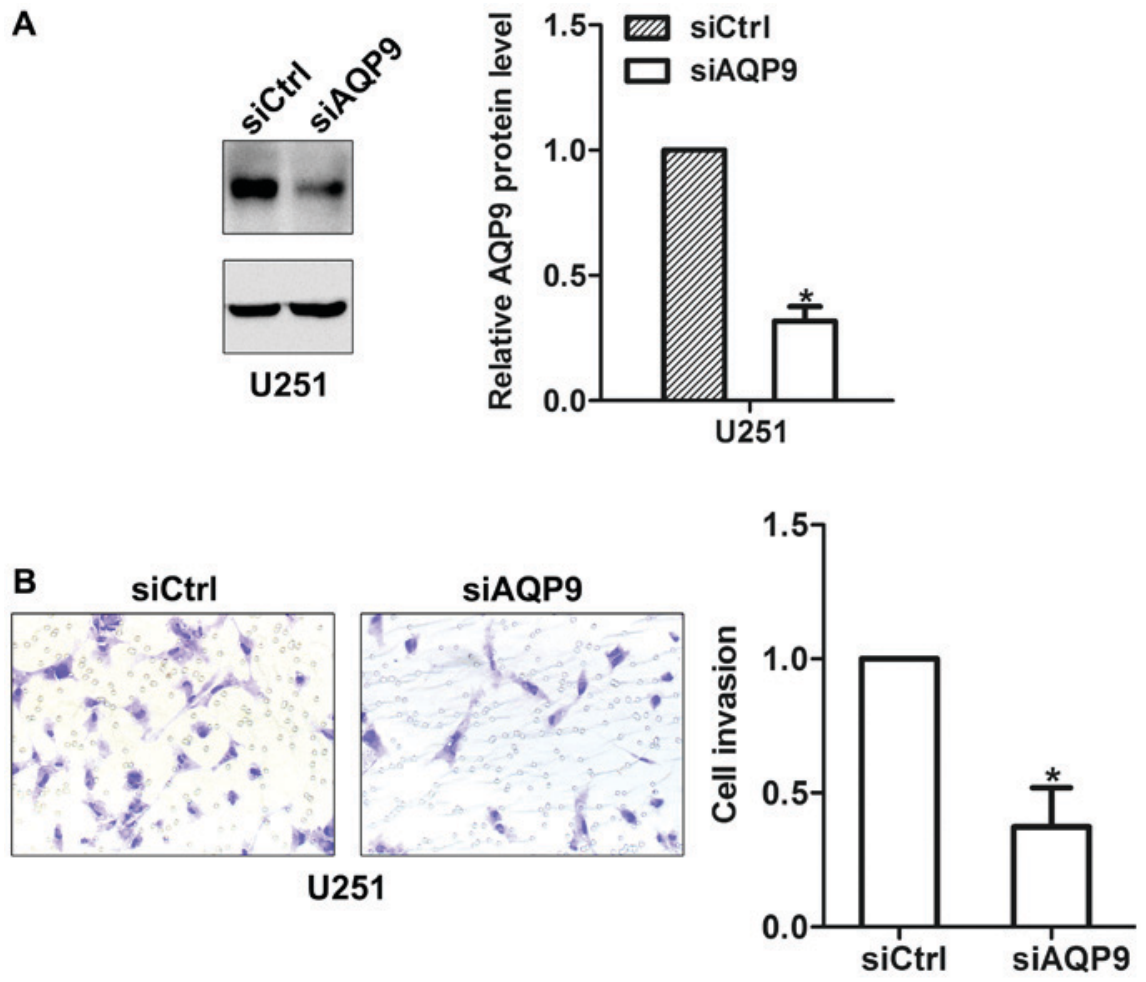

Figure 2. Effect of AQP9-knockdown on astrocytoma cell invasion. AQP9 expression in U251 cells was silenced via transfection with siAQP9. (A) Knockdown efficiency of AQP9 in U251 cells was detected by western blot analysis. (B) Effect of AQP9 knockdown on U251 cell invasion. "P<0.05. AQP9, aquaporin 9; siAQP9, small interfering RNA targeting AQP9; Ctrl, control.
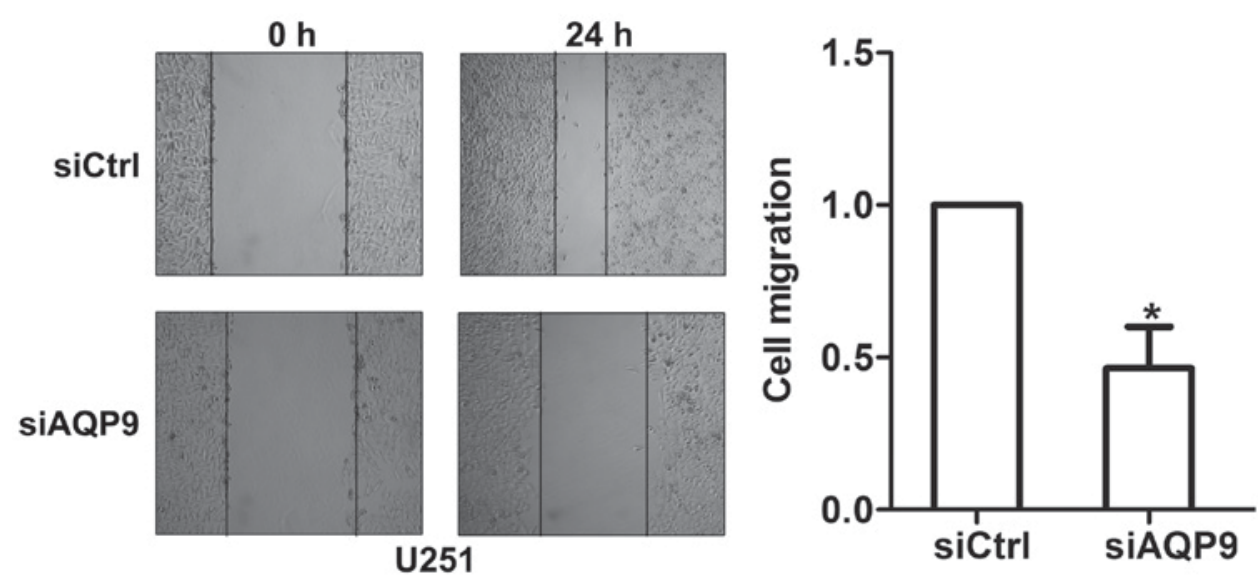

Figure 3. Effect of AQP9 knockdown on astrocytoma cell motility. "P<0.05. AQP9, aquaporin 9; siAQP9, small interfering RNA targeting AQP9; Ctrl, control.

The results of this analysis revealed that AQP9 mRNA was expressed in the two cell lines (Fig. 1A). Using western blot analysis, it was found that the AQP9 protein was expressed in SHG44 and U251 astrocytoma cells (Fig. 1B).

Knockdown of AQP9 suppresses astrocytoma cell invasion. siAQP9 was transfected into U251 cells, and the expression of AQP9 in siCtrl and siAQP9 cells was detected by western blot analysis. The results revealed that the knockdown efficiency could achieve almost $80 \%$ in U251 cells (Fig. 2A). To investigate the function of AQP9 in astrocytoma cell invasion, cell invasion assay was performed in U251 cells with or without AQP9 siRNA transfection. The results revealed that the knockdown of AQP9 markedly suppressed the invasion of
U251 cells (Fig. 2B), indicating that AQP9 has a notable role in astrocytoma cell invasion.

Knockdown of AQP9 suppresses astrocytoma cell motility. Next, following knockdown of AQP9, U251 cells were subjected to a wound-healing assay. The results of this experiment revealed that knockdown of AQP9 inhibited the motility of U251 cells (Fig. 3).

Upregulation of AQP9 promotes the invasion and motility of astrocytoma cells. To further assess the role of AQP9 in astrocytoma cell invasion and motility, SHG44 cells were transfected with pcAQP9 to increase AQP9 expression. Western blot analysis revealed that transfection with pcAQP9 
A

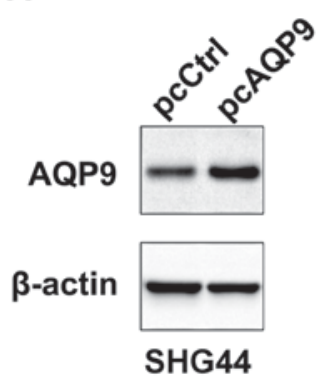

B

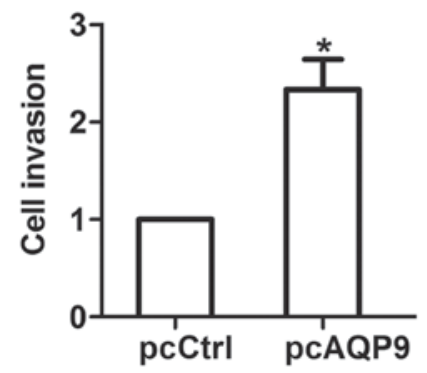

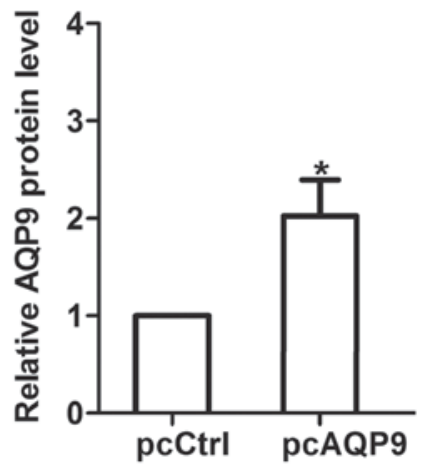

C

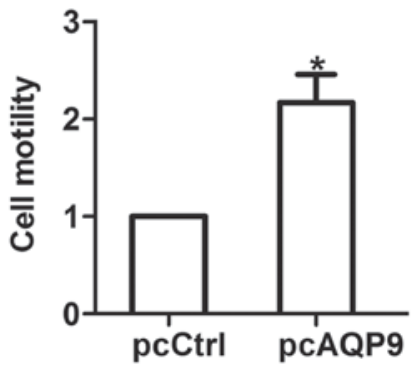

Figure 4. Effect of AQP9 overexpression on astrocytoma cell invasion and motility. AQP9 expression in SHG44 cells was upregulated by transfection with pcAQP9. (A) AQP9 expression in SHG44 cells was observed by western blot analysis. (B) Effect of AQP9 overexpression on SHG44 cell invasion. (C) Effect of AQP9 overexpression on SHG44 cell motility. ${ }^{*} \mathrm{P}<0.05$. AQP9, aquaporin 9; pcCtrl, control plasmid.

greatly enhanced AQP9 protein expression in SHG44 cells (Fig. 4A). Using invasion and wound-healing assays, it was revealed that the upregulation of AQP9 promoted the invasion and motility of SHG44 cells (Fig. 4B and C), confirming that AQP9 is essential for astrocytoma cell invasion and motility.

AQP9 induces AKT activation and decrease E-cadherin expression. The PI3K/AKT pathway has a notable role in tumor progression (12). Knockdown of AQP9 suppressed the activation of AKT in U251 cells (Fig. 5A), indicating that AQP9 can affect AKT activation in astrocytoma cells. E-cadherin is critical for tumor invasion and metastasis. Using western blot analysis, it was found that E-cadherin expression was upregulated in siAQP cells compared with siCtrl cells (Fig. 5B), indicating that AQP9 inhibits the expression of E-cadherin in astrocytoma cells.

Inhibition of AKT pathway suppresses AQP9-mediated astrocytoma cell invasion and motility. To investigate the role of the AKT pathway in AQP9-mediated cell invasion and motility, LY294002, a PI3K inhibitor (10 $\mu \mathrm{M})$, was used to suppress the PI3K/AKT pathway. Western blot analysis demonstrated that overexpression of AQP9 induced AKT activation (Fig. 6A). The invasion and wound-healing assays demonstrated that overexpression of AQP9 enhanced the invasion and motility of SHG44 cells, whereas inhibition of AKT activation attenuated AQP9-mediated astrocytoma cell invasion and motility (Fig. 6B and C). Additionally, overexpression of AQP9 decreased E-cadherin in SHG44 cells, but blockage of AKT activation attenuated the AQP9-mediated
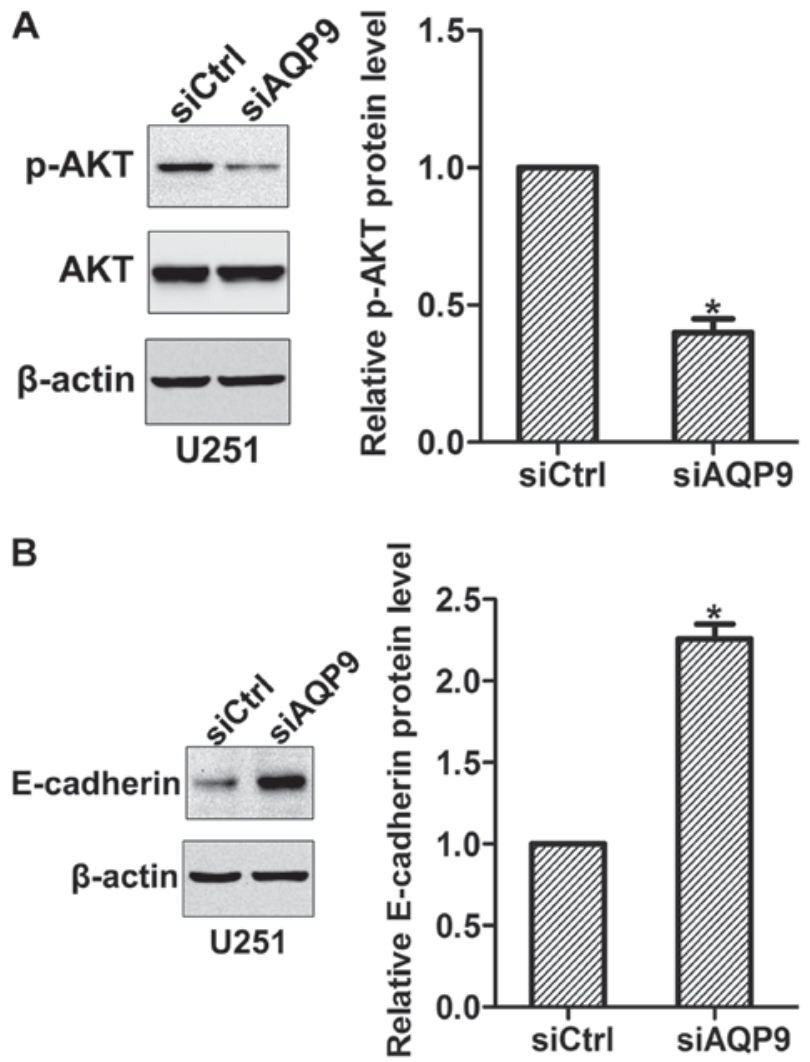

Figure 5. Effect of AQP9-knockdown on AKT activation and E-cadherin expression in astrocytoma cells. (A) Effect of AQP9 knockdown on AKT activation in U251 cells. (B) Effect of AQP9 knockdown on E-cadherin expression in U251 cells. ${ }^{*} \mathrm{P}<0.05$. AQP9, aquaporin 9; p-AKT, phosphorylated RAC serine/threonine-protein kinase; siCtrl, control small interfering RNA.

E-cadherin decrease (Fig. 6D). These data indicated that AKT pathway is involved in AQP9-mediated astrocytoma cell invasion and motility.

\section{Discussion}

AQP9 has been reported to serve a pivotal role in the development of brain tumors (13). Increases in anti-AQP9 immunoreactivity have been observed in glioma tissues, whereas in the normal brain, AQP9 is only weakly expressed by astrocytes (9). However, the molecular mechanisms of AQP9 activity in astrocytoma are unclear. The present study revealed that AQP9 was expressed in the detected astrocytoma cells, and AQP9 contributed to astrocytoma cell invasion and motility. Increases or decreases in AQP9 expression have been detected in different tumor types. AQP9 is significantly enhanced in prostate cancer tissues than that in adjacent cancer tissues (14); however, AQP9 levels are reduced in human hepatocellular carcinoma compared with the paratumoral normal liver tissues (15). The present study demonstrated that AQP9 was expressed in astrocytoma cells, indicating that AQP9 may be involved in astrocytoma cell progression.

It is reported that AQP9 contributes to cell growth and invasion of prostate cancer cells (14). However, AQP9 can suppress hepatoma cell invasion in vitro and xenograft tumor growth in vivo (16). The present study investigated the effects of AQP9 on astrocytoma cell invasion and motility. Knockdown 
A
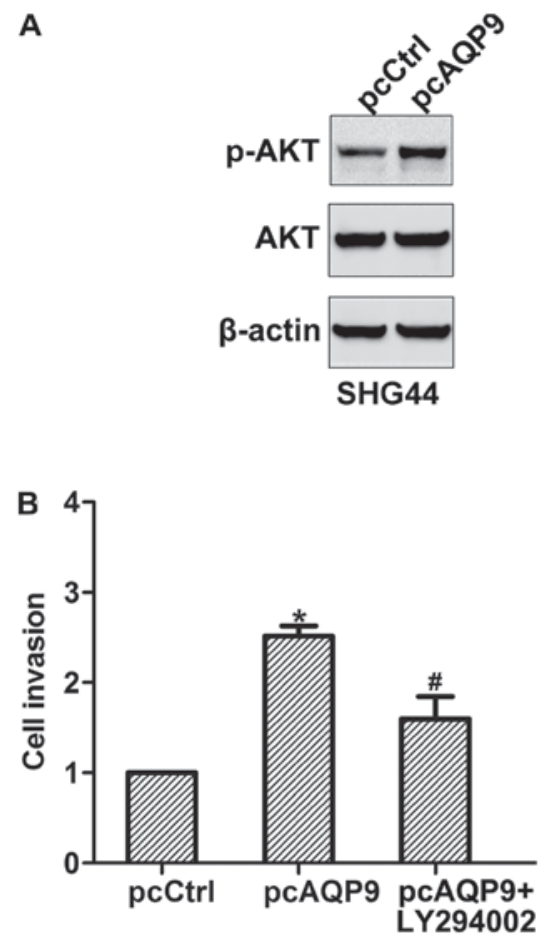

D

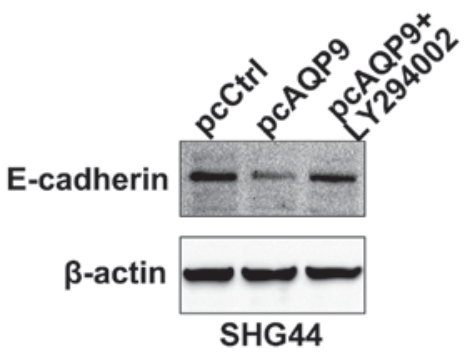

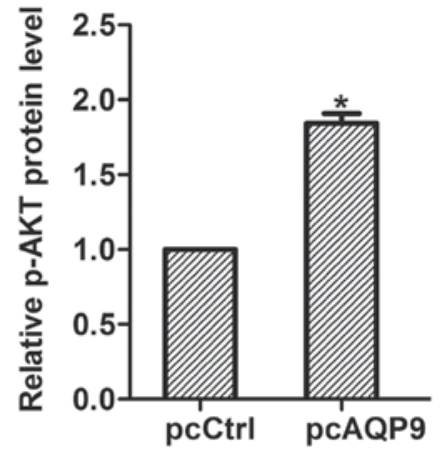
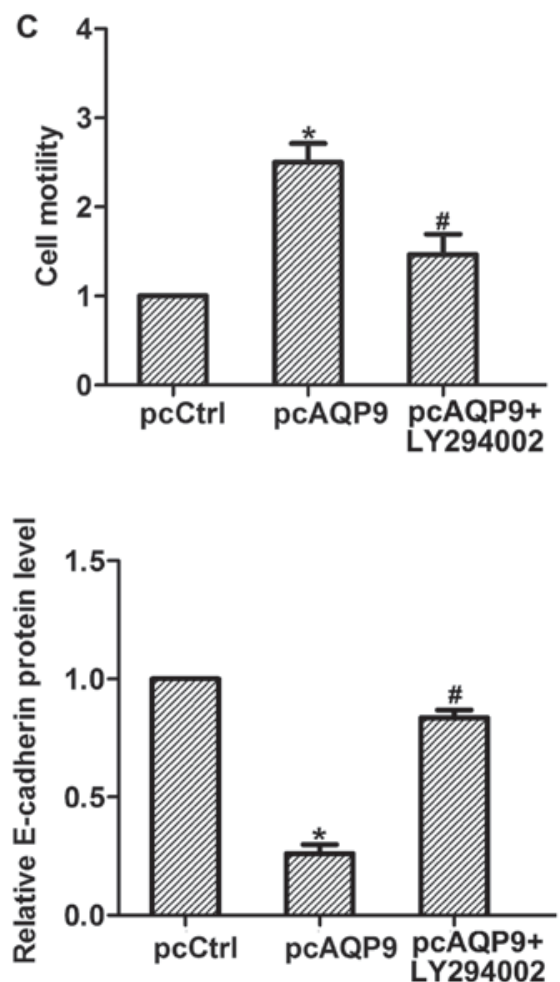

Figure 6. Effect of the AKT pathway in AQP9-mediated cell invasion and motility. (A) Effect of AQP9 overexpression on AKT activation in SHG44 cells. "P<0.05 vs. pcCtrl. (B and C) Effect of AQP9 overexpression as well as AKT inhibition on the (B) invasion and (C) motility of SHG44 cells. (D) Effect of AQP9 overexpression as well as AKT inhibition on E-cadherin expression of SHG44 cells. " $\mathrm{P}<0.05$ vs. pcCtrl; " $\mathrm{P}<0.05$ vs. pcAQP9. AQP9, aquaporin 9; p-AKT, phosphorylated RAC serine/threonine-protein kinase; pcCtrl, control plasmid.

of AQP9 suppressed astrocytoma cell invasion and motility, whereas overexpression of AQP9 promoted cellular invasion and motility. These data indicated that AQP9 has a positive role in astrocytoma cell invasion and motility.

Dysregulation of the PI3K/AKT pathway has been implicated in a number of tumor types (17). AQP3 can induce the activation of PI3K/AKT pathway in human gastric carcinoma cells (18). The role of AQP9 in AKT pathway is not clear. The present study revealed that knockdown of AQP9 reduced the activation of AKT in astrocytoma cells, whereas overexpression of AQP9 induced AKT activation. Inhibition of AKT activation attenuated astrocytoma cell invasion and motility, indicating that the AKT pathway is involved in the AQP9-mediated invasion and motility.

E-cadherin is a tumor suppressor that mediates cell-cell adhesion and cell-extracellular matrix interactions (19). Li et al (20) revealed that the downregulation of AQP4 results in increased expression of E-cadherin in breast cancer cells, although the association between AQP9 and E-cadherin expression is unclear. The present study revealed that knockdown of AQP9 increased E-cadherin expression in astrocytoma cells, whereas overexpression of AQP9 decreased E-cadherin expression. The inhibition of AKT activation suppressed the AQP9-mediated E-cadherin decrease in astrocytoma cells.

In conclusion, the data from the present study demonstrated that AQP9 was expressed in astrocytoma cells. AQP9 could induce AKT activation, decrease E-cadherin expression and promote astrocytoma cell invasion and motility. Further study may be required to investigate the effect of AQP9 on tumor metastasis in vivo.

\section{Acknowledgements}

Not applicable.

\section{Funding}

No funding received. 


\section{Availability of data and materials}

The datasets used and/or analyzed during the current study are available from the corresponding author on reasonable request.

\section{Authors' contributions}

YL and QM conceived and designed the project; YJ, GY and XF acquired the data; YL, QH and WD analyzed and interpreted the data; AW analysed the data; YL, AW and QM wrote the manuscript.

\section{Ethics approval and consent to publish}

Not applicable.

\section{Consent for publication}

Not applicable.

\section{Competing interests}

The authors declare that they have no competing interests.

\section{References}

1. Siegel RL, Miller KD and Jemal A: Cancer statistics, 2016. CA Cancer J Clin 66: 7-30, 2016.

2. Paw I, Carpenter RC, Watabe K, Debinski W and Lo HW: Mechanisms regulating glioma invasion. Cancer Lett 362: 1-7, 2015.

3. Agre P, King LS, Yasui M, Guggino WB, Ottersen OP, Fujiyoshi Y, Engel A and Nielsen S: Aquaporin water channels-from atomic structure to clinical medicine. J Physiol 542: 3-16, 2002.

4. Pelagalli A, Squillacioti C, Mirabella N and Meli R: Aquaporins in health and disease: An overview focusing on the gut of different species. Int J Mol Sci 17: pii: E1213, 2016.

5. Shi YH, Chen R, Talafu T, Nijiati R and Lalai S: Significance and expression of aquaporin 1, 3,8 in cervical carcinoma in xinjiang uygur women of China. Asian Pac J Cancer Prev 13: 1971-1975, 2012.
6. Maugeri R, Schiera G, Di Liegro CM, Fricano A, Iacopino DG and Di Liegro I: Aquaporins and brain tumors. Int J Mol Sci 17: pii: E1029, 2016

7. Chen J, Wang Z, Xu D, Liu Y and Gao Y: Aquaporin 3 promotes prostate cancer cell motility and invasion via extracellular signal-regulated kinase 1/2-mediated matrix metalloproteinase-3 secretion. Mol Med Rep 11: 2882-2888, 2015.

8. Ding T, Ma YJ, Li WL, Liu X, Ying G, Fu L and Gu F: Role of aquaporin-4 in the regulation of migration and invasion of human glioma cells. Int J Oncol 38: 1521-1531, 2011

9. Warth A, Mittelbronn M, Hulper P, Erdlenbruch B and Wolburg H: Expression of the water channel protein aquaporin-9 in malignant brain tumors. Appl Immunohistochem Mol Morphol 15: 193-198, 2007.

10. Tan G, Sun SQ and Yuan DL: Expression of the water channel protein aquaporin-9 in human astrocytic tumours: Correlation with pathological grade. J Int Med Res 36: 777-782, 2008.

11. Livak KJ and Schmittgen TD: Analysis of relative gene expression data using real-time quantitative PCR and the 2(-Delta Delta C(T)) method. Methods 25: 402-408, 2001.

12. Chautard E, Ouédraogo ZG, Biau J and Verrelle P: Role of Akt in human malignant glioma: From oncogenesis to tumor aggressiveness. J Neurooncol 117: 205-215, 2014.

13. Fossdal G, Vik-Mo EO, Sandberg C, Varghese M, Kaarbø M, Telmo E, Langmoen IA and Murrell W: Aqp 9 and brain tumour stem cells. ScientificWorldJournal 2012: 915176, 2012.

14. Chen Q, Zhu L, Zheng B, Wang J, Song X, Zheng W, Wang L, Yang D and Wang J: Effect of AQP9 expression in androgen-independent prostate cancer cell PC3. Int J Mol Sci 17: pii:E738, 2016.

15. Li CF, Zhang WG, Liu M, Qiu LW, Chen XF, Lv L and Mei ZC: Aquaporin 9 inhibits hepatocellular carcinoma through up-regulating FOXO1 expression. Oncotarget 7: 44161-44170, 2016.

16. Zhang WG, Li CF, Liu M, Chen XF, Shuai K, Kong X, Lv L and Mei ZC: Aquaporin 9 is down-regulated in hepatocellular carcinoma and its over-expression suppresses hepatoma cell invasion through inhibiting epithelial-to-mesenchymal transition. Cancer Lett 378: 111-119, 2016.

17. Toren $\mathrm{P}$ and Zoubeidi A: Targeting the PI3K/Akt pathway in prostate cancer: Challenges and opportunities (review). Int J Oncol 45: 1793-1801, 2014.

18. Xu H, Xu Y, Zhang WJ, Shen LZ, Yang L and Xu ZK: Aquaporin-3 positively regulates matrix metalloproteinases via PI3K/AKT signal pathway in human gastric carcinoma SGC7901 cells. J Exp Clin Cancer Res 30: 86, 2011.

19. Wong TS, Gao W and Chan JY: Transcription regulation of E-cadherin by zinc finger E-box binding homeobox proteins in solid tumors. Biomed Res Int 2014: 921564, 2014.

20. Li YB, Sun SR and Han XH: Down-regulation of AQP4 inhibits proliferation, migration and invasion of human breast cancer cells. Folia Biol (Praha) 62: 131-137, 2016. 